\title{
Risk-adapted graft-versus-host disease prophylaxis with post-transplantation cy- clophosphamide in related, unrelated and haploidentical stem cell transplantations
}

Olga V. Pirogova, Ivan S. Moiseev, Alexander L. Alyanski, Elena V. Babenko, Elena I. Darskaya, Olga A. Slesarchuk, Sergey N. Bondarenko, Boris V. Afanasyev

R.M. Gorbacheva Memorial Institute of Children Hematology, Oncology and Transplantation, First St.Petersburg I.P. Pavlov State Medical University, St.Petersburg, Russian Federation

Contact: Dr. Olga V.Pirogova

E-mail: Dr.Pirogova@gmail.com

\section{Introduction}

There is a growing evidence of safety and efficacy of post-transplantation cyclophosphamide (PTCy) in related and haploidentical bone marrow (BM) transplantations, but the data regarding unrelated and peripheral blood stem cell (PBSC) transplants is limited. Hence, we conducted a prospective trial of risk-adapted graft-versus-host disease (GVHD) prophylaxis with PTCy that included different types of donors and graft sources.

\section{Patients and methods}

200 adult patients (median age 32 y.o., range 18-62) with hematologic malignancies, including AML (47.5\%), ALL (26.5\%), CML (12\%), MDS (4\%), and lymphomas (10\%), were enrolled into NCT02294552 trial. 23\% of patients were classified as salvage. $26 \%$ received the graft from matched related (MRD), 65\% from matched/mismatched unrelated (MUD/MMUD), and 9\% from haploidentical (haplo) donor. 43\% received BM graft and 57\%- PBSC graft. 18.5\% had myeloablative conditioning and $81.5 \%$ - reduced-intensity conditioning. GVHD prophylaxis for matched BM graft consisted of single-agent PTCy $50 \mathrm{mg} / \mathrm{kg}$ days $+3,+4$; for matched PBSC graft we used PTCy+ tacrolimus+ mycophenolate mofetil (MMF) $30 \mathrm{mg} / \mathrm{kg}$ days 5-35, and for any mismatched graft, PTCy+ tacrolimus+ MMF $45 \mathrm{mg} / \mathrm{kg}$ at days 5-35. Median follow-up was 17 months (range 3-38).

\section{Results}

Grade II-IV, III-IV acute GVHD, and moderate-severe chronic GVHD in MRD, MUD/MMUD and haplo groups were respectively $8 \%(95 \%$ CI $3-21 \%$ ) vs $18 \%$ ( $95 \%$ CI $13-26 \%$ ) vs $26 \%$ (95\% CI $11-61 \%), \mathrm{p}=0.16 ; 4 \%(95 \%$ CI $1-16 \%)$ vs $6 \%(95 \%$ CI $3-12 \%)$ vs $0 \%, \mathrm{p}=0.57 ; 15 \%$ (95\% CI 7-29\%) vs $10 \%$ (95\% CI $5-17 \%)$ vs $16 \%$ (95\% CI 5-59\%), $\mathrm{p}=0.35$. Non-relapse mortality (NRM) and relapse incidence were $8 \%(95 \%$ CI $3-22 \%)$ vs $14 \%$ (95\% CI $9-21 \%$ ) vs $16 \%$ (95\% CI 6- $45 \%$ ), $\mathrm{p}=0.50$, and $38 \%$ (95\% CI 30 $66 \%$ ) vs $23 \%$ (95\% CI $16-32 \%$ ) vs $45 \%$ (95\% CI $27-76 \%$ ), $\mathrm{p}=0.02$ for MRD, MUD/MMUD and haplo group, respectively. 2-year overall survival (OS), event-free-survival (EFS), and GVHD-relapse free survival (GFRS) were $70 \%$ (95\% CI $53-82 \%$ ) vs $67 \%$ ( $95 \%$ CI $57-75 \%$ ) vs $44 \%$ (95\% CI 21-65\%), $\mathrm{p}=0.007$; $53 \%$ (95\% CI $38-67 \%$ ) vs $64 \%$ (95\% CI $54-72 \%$ ) vs $36 \%$ (95\% CI 15-57\%), $\mathrm{p}=0.009 ; 37 \%$ (95\% CI $23-51 \%)$ vs $59 \%(95 \%$ CI $50-67 \%)$ vs $36 \%$ (95\% CI $15-57 \%$ ), $\mathrm{p}=0.035$ for MRD, MUD/MMUD and haplo groups, respectively. There were no statistical differences in OS, EFS and GFRS between MUD and MMUD ( $p>0.05$ ). In a multivariate analysis, only salvage status (HR 3.0 95\%CI 1.94.7, $\mathrm{p}<0.0001$ ) and occurrence of sepsis (HR $1.795 \% \mathrm{CI}$ 1.0-2.7, $\mathrm{p}=0.04$ ) were predictive for $\mathrm{EFS}$, while type of donor was not a significant factor (HR 1.1 95\%CI 0.7-1.9, $\mathrm{p}=0.60$ ) (Fig. 1). The incidences of complications were: hemorrhagic cystitis, 23\%; sepsis, $24 \%$; severe sepsis, $8 \%$; invasive mycosis, $8 \%$; CMV reactivation $45 \%$; veno-occlusive disease, $2.5 \%$; transplant-associated microangiopathy, $3.5 \%$; grade $3 / 4$ liver toxicity, $14 \%$; grade $3 / 4$ kidney toxicity, $1 \%$. 


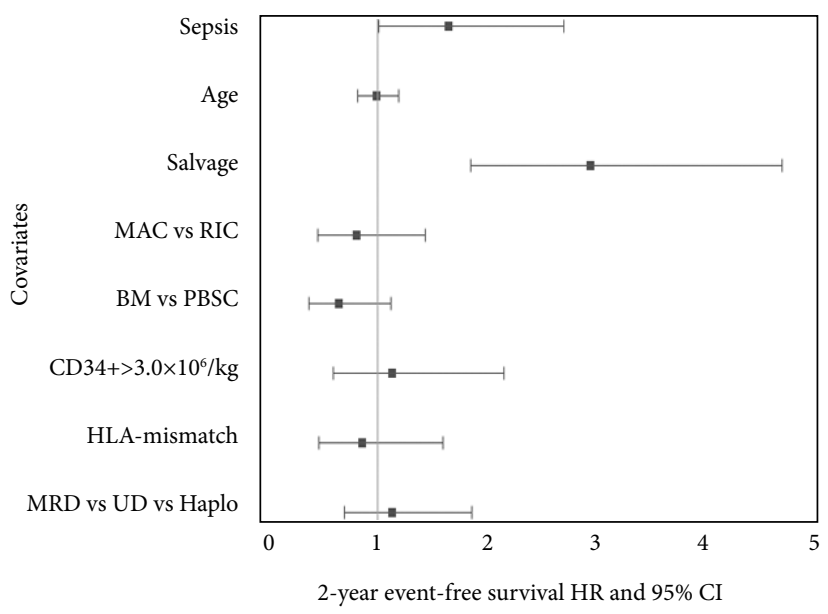

Figure 1. Multivariate analysis of event-free survival

\section{Conclusion}

With relatively short follow-up, we have demonstrated that the risk-adapted PTCy GVHD prophylaxis is safe and results in very low incidences of NRM, acute and chronic GVHD. Therefore, it could be used with any kind of graft source. It also alleviates the GVHD risk in MMUD and haplo donor settings. Relapse of underlying malignancy still significantly influences the outcome with this mode of prophylaxis.

\section{Keywords}

Allogeneic hematopoietic stem cell transplantation, graftversus-host disease (GvHD), prophylaxis, cyclophosphamide.

\section{Риск-адаптированная стратегия профилактики реакции трансплантат-против-хозяина на основе посттрансплантационного циклофосфана при родственных, неродственных и гаплоидентичных трансплантациях}

Ольга В. Пирогова, Иван С. Моисеев, Александр Л. Алянский, Елена В. Бабенко, Е. И. Дарская, Ольга А. Слесарчук, Сергей Н. Бондаренко, Борис В. Афанасьев

НИИ детской онкологии, гематологии и трансплантоллогии им. Р.М. Горбачевой, Первый Санкт-Петербургский государственный медицинский университет им. И.П. Павлова

\section{Введение}

В настоящее время растет количество сообщений о безопасности и эффективности посттрансплантационного циклофосфана (ПТЦи) при гаплоидентичных и родственных трансплантациях костного мозга (КМ). Применимость этого метода при других видах трансплантации гемопоэтических стволовых клеток (ТГСК) неизвестна. Цель работы: Оценить эффективность риск-адаптивной стратегии профилактики реакции трансплантат-против-хозяина (РТПХ) с использованием ПТЦи при полностью и частично HLA-совместимых ТГСК и различных источниках трансплантации.

\section{Материалы и методы}

200 взрослых пациентов (медиана возраста 32 года, диапазон 18-62) с гемобластозами, включая ОМЛ $(47,5 \%)$, ОЛЛ $(26,5 \%)$, ХМЛ (12\%), МДС (4\%) и лимфомы (10\%), были включены в проспективное исследование (clinicaltrials.gov №NCT02294552). 26\% пациентов была выполнена родственная (РТ), 65\% неродственная (НРТ), и 9\% - гаплоидентичная (Гапло) ТГСК. 43\% была выполнена трансплантация КМ, 57\% - трансплантация периферических стволовых клеток (СКПК). 18,5\% проведено миелоаблативное кондиционирование и $81,5 \%$ со сниженной токсичностью. Профилактика РТПХ для совместимого КМ проводилась монотерапией циклофосфаном 50 мг/кг в дни+3,+4, для совместимых СКПК - ПТЦи + такролимус+ микофенолата мофетил (MMF) 30 мг/кг в дни $+5-35$, а для частично совместимой ТГСК - ПТЦи + такролимус+MMF - 4530 мг/кг в дни +5-35. Медиана наблюдения составила 17 месяцев (диапазон 3-38).

\section{Результаты}

Частота острой РТПX II-IV, III-IV степени и средней + тяжелой хронической РТПХ составили 8\% (95\% CI $3-21 \%$ ) vs $18 \%$ (95\% CI $13-26 \%$ ) vs $26 \%$ (95\% CI $11-61 \%$ ), $\mathrm{p}=0.16 ; 4 \%(95 \%$ CI $1-16 \%)$ vs $6 \%(95 \%$ CI $3-12 \%)$ vs $0 \%$, $\mathrm{p}=0.57 ; 15 \%(95 \%$ CI $7-29 \%)$ vs $10 \%$ (95\% CI 5-17\%) vs $16 \%$ (95\% CI 5-59\%), $\mathrm{p}=0.35$ для родственных, неродственных 
и гаплоидентичных ТГСК. Трансплантационная летальность и частота рецидивов составили 8\% (95\% CI 3-22\%) vs $14 \%$ (95\% CI $9-21 \%$ ) vs $16 \%$ (95\% CI 6-45\%), $\mathrm{p}=0.50$, and $38 \%$ (95\% CI $30-66 \%$ ) vs $23 \%$ (95\% CI $16-32 \%$ ) vs $45 \%$ (95\% CI 27-76\%), p=0.02 для РТ, НРТ и Гапло групп, соответственно. 2-летняя общая выживаемость (OB), бессобытийная выживаемость (БСВ), и выживаемость без рецидива и РТПХ (ВРРТПХ) составили 70\% (95\% СІ 53-82\%) vs $67 \%$ (95\% CI $57-75 \%$ ) vs $44 \%$ (95\% CI $21-65 \%$ ), $\mathrm{p}=0.007$; $53 \%$ (95\% CI $38-67 \%)$ vs $64 \%$ (95\% CI $54-72 \%)$ vs $36 \%(95 \%$ CI $15-57 \%), \mathrm{p}=0.009 ; 37 \%$ (95\% CI $23-51 \%)$ vs $59 \%(95 \%$ CI $50-67 \%$ ) vs $36 \%$ (95\% CI 15-57\%), $\mathrm{p}=0.035$ для РТ, НРТ и Гапло групп, соответственно. Показатели ОВ, БСВ, ВРРТПХ достоверно не различались между полностью и частично совместимой НРТ ( $>0.05)$. В многофакторном анализе только принадлежность к группе «терапии спасения» (ОР 3.0 95\% ДО ОР 1.9-4.7, р<0.0001) и развитие сепсиса (ОР 1.7 95\% ДО ОР 1.0-2.7, p=0.04) влияли на бессобытийную выживаемость, в то время как тип донора не был достоверным фактором (ОР 1.1 95\%ДО ОР 0.7-1.9, p=0.60) (рис 1). Частота осложнений составила: геморрагический цистит - $23 \%$, сепсис - $24 \%$, тяжелый сепсис -8\%, инвазивный аспергиллез - 8\%, ЦМВ-реактивация $-45 \%$, вено-окклюзионная болезнь $-2.5 \%$, трансплантационная микроангиопатия - 3.5\%, печеночная токсичность 3-4 степени - 14\%, почечная токсичность 3-4 степени - $1 \%$.

\section{Заключение}

При относительно небольшом времени наблюдения, наши предварительные результаты говорят об эффективности и безопасности риск-адаптированной профилактики РТПХ с ПТЦи для всех типов доноров и источников трансплантата.

\section{Ключевые слова}

Аллогенная трансплантация гемопоэтических стволовых клеток, болезнь «трансплантат против хозяина», профилактика, циклофосфамид. 